\title{
Entrelacs
}

Cinéma et audiovisuel

HS | 2008

Séries télé in/out

\section{Réception et réécriture : les fanfics de The Shield}

\section{Miguel-Angel Torres}

\section{OpenEdition}

\section{Journals}

Édition électronique

URL : http://journals.openedition.org/entrelacs/266

DOI : 10.4000/entrelacs.266

ISSN : 2261-5482

Éditeur

Éditions Téraèdre

\section{Édition imprimée}

Date de publication : 7 février 2008

ISSN : 1266-7188

Référence électronique

Miguel-Angel Torres, «Réception et réécriture : les fanfics de The Shield », Entrelacs [En ligne], HS | 2008, mis en ligne le 01 août 2012, consulté le 20 avril 2019. URL : http://

journals.openedition.org/entrelacs/266; DOI : 10.4000/entrelacs.266

Ce document a été généré automatiquement le 20 avril 2019.

Tous droits réservés 


\title{
Réception et réécriture : les fanfics de The Shield
}

\author{
Miguel-Angel Torres
}

1 Le terme fanfiction renvoie à la pratique de création de fictions par des fans des séries télévisées, des films, etc. Les fans qui écrivent des fanfics sont ainsi des personnes qui ont forgé dans le temps un sentiment d'attachement au texte et qui, ultérieurement, ont produit elles-mêmes des fictions basées sur ces univers diégétiques.

2 Notre travail prendra les fanfics sous forme de récits écrits de The Shield comme élément d'analyse des stratégies réinterprétatives et de réécriture de fans. Selon une approche sémiotique, nous confronterons la série aux fanfics produits à partir d'elle. Notre objectif sera de voir à quel degré les auteurs redessinent les représentations présentes dans la série. A cet égard, ce travail fera le choix de se centrer sur la comparaison de ces deux textualités, laissant de côté d'autres aspects certainement importants pour l'analyse de la réception.

3 Notre analyse de fanfics de The Shield se trouve confrontée à la limitation d'un fandom moins grand et moins actif que celui de la Science Fiction (à ce sujet, voir JENKINS, 1992 ; STAIGER, 2006). En nous imposant la limite de travailler avec les fanfics produits entre le début de la diffusion de la série et la fin de la deuxième saison, pour mieux maîtriser la comparaison entre la série diffusée et les fanfics écrits à partir d'elle, nous avons eu affaire à un nombre de cinq auteurs pour trente fanfics. Les fanfics ont été recueillis sur un site dédié à la fanfiction : Fanfiction.net. Parmi les différents sites consacrés aux fanfics écrits, Fanfiction.net est celui qui montre le plus d'activité. Il est aussi le seul à avoir des fanfics de The Shield, hébergeant au moment de notre recherche - mars 2006 - 115 fanfics.

4 The Shield est une série policière américaine produite par la chaîne du câble FX. La série se caractérise par une narration centrée sur le thème de la corruption policière. Le personnage principal, Vic Mackey, et l'équipe anti-gang qu'il dirige (la Strike Team) sont présentés, surtout dans les trois premières saisons, comme des policiers violents et corrompus. Bien que la série développe aussi des récits policiers classiques - des enquêtes policières -, l'arc narratif de la saison s'articule autour du thème de la corruption, du danger pour Strike Team de tomber à cause de ses activités illicites. 
5 La forme narrative de la série présente ainsi deux lignes narratives constantes dans tous ses saisons : une grande ligne narrative articulant la saison - Vic Mackey confronté aux efforts du capitaine Aceveda pour le faire tomber - et le récit des enquêtes policières souvent résolues dans chaque épisode par les personnages de Dutch et Claudette. La narration est situé dans un espace et un temps précis : l'actualité, dans une banlieue marginale de Los Angeles. La formule de la série est complétée par ces constantes sémantiques : une représentation qui se veut réaliste de la banlieue de Los Angeles, ce qui amène à présenter des communautés latinos et noires. Puisque la représentation des pouvoirs publics et de la corruption est déjà présente dans la série, il était aussi de notre intérêt de voir si les fanfics allaient plus loin dans cette représentation critique des policiers. D'une manière générale, l'objectif était de voir comment la formule de la série, qu'on vient de décrire brièvement, avait était reprise par les auteurs de fanfics.

\section{Formes narratives des fanfics}

6 Si on prend la notion de récit dans le sens greimassien où, pour qu'il y ait récit, il faut avoir affaire à une transformation, au passage d'un État 1 à un État 2 (COURTES, 1991 : 70), on remarque que tous les fanfics de notre corpus ne rentrent pas dans cette définition. Alors que certains racontent vraiment des récits, d'autres ne font que s'arrêter sur la description des états. Notre premier tableau intègre à cette distinction, un regroupement des récits d'après leur obéissance à la formule narrative de la série (les deux lignes narratives décrites) :

Tableau 1

\begin{tabular}{|c|c|c|}
\hline Fanfics décrivant des états & $\begin{array}{l}\text { Fanfics en forme de récits } \\
\text { suivant la formule de la } \\
\text { série }\end{array}$ & $\begin{array}{l}\text { Fanfics en forme de récits ne } \\
\text { suivant pas la formule de la } \\
\text { série }\end{array}$ \\
\hline $\begin{array}{l}\text { 1.«Sinchronicity } 1 » \quad \text { par } \\
\text { Katt9966 }\end{array}$ & 1. « Flames » par LeaO'Neill & $\begin{array}{l}\text { 1. «Tuesday's Gone» par } \\
\text { LeaO'Neill }\end{array}$ \\
\hline $\begin{array}{l}\text { 2. «Sinchronicity 2» par } \\
\text { Kat9966 }\end{array}$ & 2. « Torn » par LeaO’Neill & $\begin{array}{l}\text { 2. «Look Away» par } \\
\text { LeaO'Neill }\end{array}$ \\
\hline 3. «Soulmates » par Kat9966 & 3. «Justice » par LeaO’Neill & $\begin{array}{l}\text { 3. «Sing the Blues» par } \\
\text { LeaO'Neill }\end{array}$ \\
\hline $\begin{array}{l}\text { 4. «No one wants to die» par } \\
\text { OriginalWWEMamacita }\end{array}$ & & $\begin{array}{l}\text { 4. «When I'm gone» par } \\
\text { LeaO'Neill }\end{array}$ \\
\hline 5. « Mismatch $1 »$ par Katt9966 & & 5. « Night » par Katt9966 \\
\hline 6. « Mismatch $2 »$ par Katt9966 & & 6. « Trespass » par Katt9966 \\
\hline 7. « Alternatives » par Katt9966 & & 7. « Friendship » par Katt9966 \\
\hline 8. « Daydream » par Katt9966 & & 8. « Identity » par Katt9966 \\
\hline
\end{tabular}




\begin{tabular}{|c|c|}
\hline 9. « Red » par Katt9966 & $\begin{array}{l}\text { 9. «Past present» par } \\
\text { Whipper }\end{array}$ \\
\hline 10. «Blue » par Katt9966 & $\begin{array}{l}\text { 10. «Real recruit» par } \\
\text { Str8chillin }\end{array}$ \\
\hline 11. «Dawn » par Katt9966 & \\
\hline 12. « Rain Dawn » par LeaO'Neill & \\
\hline 13. «Scorned » par Katt9966 & \\
\hline 14. « Bleachers » par Katt9966 & \\
\hline 15. «Video » par Katt9966 & \\
\hline $\begin{array}{l}\text { 16. «Betrayal series» par } \\
\text { Katt9966 }\end{array}$ & \\
\hline 17. « Night out » par LeaO’Nei & \\
\hline
\end{tabular}

7 À partir de ce tableau, nous serions tentés de dire que la pratique de la création de fanfics inclut certaines formes de reproduction de la formule, mais ne se limite pas à cette répétition. L'exploration d'autres possibilités narratives est ainsi loin d'être une exception et semble plutôt être la règle.

8 Si on examine cette fois ce tableau à partir des auteurs de fanfics, on observe que des cinq auteurs, deux ont posté la plupart d'eux : LeaO'Neill (9) et Katt9966 (19). Les trois autres n'apparaissent qu'avec un fanfic chacun. Le degré inégal de participation est en fait caractéristique des petits fandoms, où il y a toujours, tout comme dans notre corpus, un petit nombre des participants très actifs et d'autres qui participent éventuellement. En fait, même si on étend la période jusqu'à nos jours, c'est toujours Katt9966 qui est la participante la plus active - la participation de LeaO'Neill s'arrête peu après la période de notre analyse ${ }^{1}$.

9 Majoritairement, les fanfics décrivant des états détaillent des situations émotionnelles de personnages et rarement des états de choses. Ainsi, un seul fanfic s'attarde sur la présentation d'un état alternatif de choses («Alternatives» par Katt9966). Notre deuxième tableau fait une distinction entre ces fanfics : soit ils explorent les émotions des personnages à un moment donné de la série, soit ils le font à un moment qui n'a pas eu lieu dans la série :

Tableau 2

\begin{tabular}{|l|l|}
\hline Etats situés dans la série & Etats situés hors de la série \\
\hline 1. « Sinchronicity 1 » par Katt9966 & 1. « No one wants to die » par Original WWEMamacita \\
\hline 2. « Sinchronicity 2 » par Katt9966 & 2. «Bleachers » par Katt9966 \\
\hline 3. « Soulmates » par Katt9966 & 3. «Alternatives » par Katt9966 \\
\hline
\end{tabular}




\begin{tabular}{|l|l|}
\hline 4. « Mismatch 1 » par Katt9966 & 4. « Red » par Katt9966 \\
\hline 5. « Mismatch 2 » par Katt9966 & 5. « Blue » par Katt9966 \\
\hline 6. « Scorned » par Katt9966 & 6. « Dawn » par Katt9966 \\
\hline 7. « Night Out » par LeaO'Neill & 7. « Daydream » par Katt9966 \\
\hline 8. « Rain Dawn » par LeaO'Neill & 8. « Video » par Katt9966 \\
\hline 9. « Betrayal series » par Katt9966 & \\
\hline
\end{tabular}

10 Dans le premier groupe, il s'agit d'arrêts sur des moments précis de la série -souvent indiqués dans le paratexte (autant pour « Sinchronicity 1 » et « Sinchronicity 2 » que pour "Mismatch 1 » et Mismatch 2 », Katt9966 les décrit comme " an episode tag for 'Cupid and Psycho'») - ou sur des situations pour lesquelles la série est passée rapidement (par exemple, la séparation entre Dutch et Kim, à peine traitée dans la série, est le sujet de «Scorned»). On se trouve ainsi face à des textes qui pourraient s'intégrer à la ligne narrative de la série sans aller contre sa cohérence. Ils sembleraient même, dans ce sens, compléter les vides du texte de base ${ }^{2}$.

Dans le deuxième groupe de notre tableau, les états auxquels on fait allusion se passent en dehors de l'univers de la série. Le centre même du texte n'est pas tant de présenter la situation que d'explorer l'état émotionnel du personnage dans cette situation critique : Ronnie croit être en train de mourir dans "No one wants to die " (écrit par OriginalWWEMamacita); Dutch, angoissé, réfléchi à sa situation après avoir été torturé et abusé dans « Dawn » (écrit par Katt9966), etc. On trouve aussi un exemple de slash (fanfic racontant une histoire homosexuel): dans Daydream de LeaO'Neill trois personnages (Shane, Vic et Ducth) ont de rapports homosexuels avec un personnage créé par l'auteur.

Dans le cas de fanfics racontant des histoires (Tableau 1) on a affaire à une situation assez intéressante, puisqu'on n'est plus dans une forme narrative qu'on ne trouve pas dans la série. Les trois fanfics qui se tiennent le plus à la formule -les trois écrits par LeaO'Neillmettent en jeu les formes narratives constitutives de la série. On voit ainsi deux lignes narratives s'entrecroiser : par exemple, dans «Flames ", tandis que Vic et la Strike team se confrontent à une bande dirigée par un ex-policier, Aceveda essaie de se servir d'un enregistrement fait par Terry Crowley ${ }^{3}$ pour faire tomber Vic Mackey ; et dans « Torn » le meurtre accidentel d'un jeune homme par shane lors d'une descente permet à David Aceveda de s'attaquer à la Strike Team. Dans ces récits, comme dans la série, Vic Mackey et son équipe finissent par se sortir des accusations. Cet attachement à la formule peut bien être considéré comme une question qui ne concerne qu'elle, puisque notre examen de la période postérieure à celle de notre corpus ne montre aucun exemple similaire.

Les récits qui s'éloignent de la formule de la série sont beaucoup plus nombreux et ne se limitent pas à un seul auteur : dix récits écrits par quatre auteurs ${ }^{4}$ (quatre de LeaO'Neill, quatre de Katt9966, un de Whipper et 1 de Str8chillin). S'il y a des constantes dans cet éloignement de la formule, ce sont le déplacement de l'attention vers des personnages secondaires et la perte de la ligne narrative définitoire de la série. On se trouve face à des récits qui se situent dans l'enquête policière, mais sans brouiller cette forme narrative 
avec celle de la confrontation Aceveda vs Mackey. On n'est plus sur le plan des manipulations qui est au centre de The Shield.

Parmi ces dix récits, il y a cinq qui ne peuvent pas s'intégrer dans la cohérence diégétique de la série : « Night », « Trespass » et «Identity » de Katt9966, « Past present » de Whipper et «Real recruit» de Str8chillin. Dans ces récits, il se déroule, par exemple, la possible mort de Dutch («Identity » de Katt9966), le kidnapping, viol et torture de Dutch par un serial killer («Night» de Katt9966), une relation homosexuel entre Vic et Dutch («Trespass » de Katt9966), etc. Si ces fanfics s'éloignent à un degré différent de l'univers de la série, ils s'appuient néanmoins sur des vides, des non-dits et des espaces ainsi ouverts dans le texte. Ainsi, après les événements dans l'épisode 'Cherrypoppers', la phrase incomplète de Dutch "quand j'étais petit...." sert de point de départ aux élaborations du fanfic de Whipper 'Past present's.

\section{Formes sémantiques et énonciatives}

15 Si l'on considère les constantes sémantiques de la série, on remarque que les textes conservent globalement une sorte d'obéissance à la série. Dès ce point de vue, la seule grande exception est constituée par les récits en forme de slash. Hormis ces cas-là, tous les autres récits montrent toujours Vic Mackey et la Strike Team franchissant la limite de ce qui est légal, arrivant même à voler les délinquants (dans « Look Away » et «Justice » de LeaO'Neill). Ce ne sont pas, donc, Dutch, Claudette, Aceveda ou Julien qui apparaissent faisant ces actes. On ne trouve dans notre corpus ni dans la période d'après un seul cas où on joue à subvertir l'organisation sémantique de la série ou à tordre son axiologie. Si exploration sémantique il y a, pouvant être comprise comme une forme de réinterprétation ou de réécriture, elle consiste à explorer les sentiments de personnages.

Les formes énonciatives privilégiées dans les fanfics semblent ainsi aller dans le sens de cette exploration : elles sont dans presque tous le cas des récits clairement focalisés sur un personnage. On est, alors, face à ce que Genette appelle la focalisation interne ; c'est-àdire les cas où le foyer de perception de la narration est situé du point de vue d'un personnage (GENETTE, $1972: 43$; Cf. aussi FONTANILLE, 1989 :17-21). Un exemple de chaque auteur de notre corpus peut montrer comment c'est une stratégie de narration majoritaire.

Katt9966: "Mismatch 1"

Christ, Shane Vendrell was an unthinking, insensitive moron. He hoped this was only for one day, because Dutch didn't think he'd last any longer without taking his stupid orange ball and ramming it down his throat. [...]

LeaO'Neill : "Justice"

Shane had been shot at, punched and generally worked over the last few days. Which was nothing new for him. But he really needed a beer. Which he was thoroughly enjoying, along with a smoke, right this moment. [...]

Str8chillin : "Real Recruit"

The alarm goes off and Vic instantly hits the snooze. The phone rings, Vic groans and rolls over to answer. "Hello !" The person on the other end is Shane, Vic's right hand man. "Vic, you awake, man ?" Vic answers with a tired voice, "Yeah, what's up ?"

Whipper: "Past Present"

He kept his eyes firmly aimed at the file in front of him although, truth to be told, he probably couldn't even give a brief summary of it's content. The words were just 
dancing around on the page, randomly changing places with each other in some, undoubtedly very evil, conspiracy to drive him insane. [...]

OriginalWWEMamacita : "No one wants to die"

I fell to the floor and I heard Lem and Shane fire their guns and heard the screams of the man who I'm guessing shot me. Pretty soon Lem and Shane rolled me over and Vic began examining me, to see how bad my injury really was.[...]

Dans «Mismatch 1 ", l'invective avec laquelle commence le texte ("Christ») et les qualifications faites sur Shane ( $[$ [...] was an unthinking, insensitive moron. ») ne peuvent être comprises que venant de l'intériorité de Dutch; dans "Justice», la possibilité de comprendre les désirs de Shane n'est possible que parce qu'on se place dans le point de vue de cet acteur de l'énoncé, etc.

S'il y a une stratégie énonciative qui est commune, c'est celle de mettre le lecteur dans une position de grande proximité avec le personnage traité, par un ancrage sur la perspective thymique et pragmatique d'un des acteurs de l'énoncé. Il y a ainsi une constante sémantique et une stratégie énonciative privilégiées dans les fanfics qui sont plutôt étrangères à la série. Là où la série met en œuvre des récits, se centre sur la thématique de la corruption et place le spectateur dans une position d'expectative pour la résolution des confrontations entre policiers, les récits sous cette forme sont loin d'être la forme privilégiée dans les textes de notre corpus.

Les traits les plus retenus par rapport aux constantes sémantiques de la série sont alors ceux de : la forme policière du texte (le genre) et la caractérisation des personnages (les traits figuratifs sous lesquels ils apparaissent) et des espaces (le commissariat et la représentation de la banlieue de Los Angeles, ce qui inclût le fait de situer les histoires dans des milieux latinos et d'utiliser de noms latinos pour les gangs).

Les fanfics qui constituent notre corpus se caractérisent par le fait qu'ils privilégient l'exploration des sentiments des personnages. Le lecteur est ainsi situé de façon à connaître les nuances émotionnelles des personnages, dès que le foyer de perception de la narration s'ancre dans le point de vue thymique et pragmatique d'un des acteurs. Il y a ainsi une volonté d'explorer plus en profondeur les motivations, contradictions et émotions de personnages, tout en gardant leurs traits figuratifs distinctifs : Dutch étant plutôt faible, Shane assez maladroite, Vic dur, etc. S'il y a, alors, un faire savoir en jeu, auquel est soumis le lecteur de ces fanfics, ce faire savoir concerne la connaissance plus profonde des sentiments et des motivations des personnages.

\section{Conclusions}

21 Notre analyse des fanfics de The Shield nous amène à une position plus nuancée que celle de ceux qui pensent au téléspectateur comme un stratège qui fait usage des contenus du texte pour les détourner ou les utiliser d'après leurs besoins (la perspective d'usages et gratifications). Nous ne considérons pas que toute forme de réappropriation des discours médiatiques, per se, constitue une forme de détournement des contenus du texte de base. Le concept de braconnage (DE CERTEAU, 1990), si souvent emprunté à De Certeau par des chercheurs en réception (JENKINS, 1992 ; FISKE, 1987 ; ANG, 1985, etc.), doit alors être utilisé avec prudence. Notre analyse à montré que le travail de réappropriation des univers diégétiques s'est fait dans notre corpus sans jamais aller jusqu'à renverser l'organisation sémantique qui caractérise la formule de la série. 
L'exploration de l'univers diégétique du texte de base est faite majoritairement sans sortir de cet univers, mais en développant plus profondément des traits de caractérisations déjà présents. Si on n'identifie pas stratégie avec stratégie subversive, on peut alors bien dire que la fanfiction témoigne d'une pratique de création stratégique, puisqu'il est en jeu en elle le rapport qu'il existe entre le fanfic et le texte de base.

\section{BIBLIOGRAPHIE}

ANG, Ien

1996 Living room wars. Rethinking media audiences for a postmodern world. Londres, Routdledge.

1985 Watching Dallas : Soap Opera and the Melodramatic Imagination. Londres, Methuen.

COURTES, Joseph

2003 La sémiotique du langage. Paris, Nathan.

1991 Analyse sémiotique du discours. Paris, Hachette.

DE CERTEAU, Michel

1990 L'invention du quotidien. 1. Arts de faire. Paris, Gallimard.

FISKE, John

1987 Television culture. Londres, Methuen.

FONTANILLE, Jacques

1989 Les espaces subjectifs. Introduction à la sémiotique de l'observateur. Paris, Hachette.

GENETTE, Gérard

1972 Figures III. Paris, Editions du Seuil.

JENKINS, Henry

1992 Textual poachers. Television fans \& participatory culture. New York, Routledge.

STAIGER, Janet

2005 Media reception studies. New York, New York University Press.

\section{NOTES}

1. De 85 fanfics non compris dans notre corpus, 52 ont été écrits par Katt9966. Dans cette période il y a 14 auteurs. Après Katt9966 ceux qui ont posté plus de fanfics sont Templeton, avec 6 fanfics, et Gsmadness avec 7 fanfics. Tous les autres n'ont posté qu'un ou deux fanfics.

2. La période postérieure à celle de constitution de notre corpus présente pas mal d'exemples de ce type de texte. Un bel exemple est « The start of something ", fanfic écrit par Templeton21, qui développe en plusieurs chapitres (19) une relation amoureuse entre Lem et Tigre, relation qui avait en fait commencée dans l'épisode 9 de la saison 1, mais qui n'avait été plus développée dans la série. Ce qui est spécial dans ce fanfic, c'est qu'il prend comme point de départ, dans chacun de ces chapitres, des moments des différentes saisons où Lem n'est pas présent. Cette volonté d'intégration avec la cohérence diégétique va, en fait, jusqu'à un chapitre récent, de la saison 5 , où le personnage de Lem meurt. 
3. Terry Crowley est le policier tué par Mackey dans l'épisode pilote.

4. On comprend dans cette liste deux histoires incomplètes : «Real recruit » par str8chillin' et «Friendship » par Katt9966.

5. Ce même épisode et l'incomplétude de la phrase de Dutch seront à la base du fanfic de Katt9966 «Video ».

\section{RÉSUMÉS}

Les séries télévisées sont au sein de fandoms sujets de discussion, d'échanges, de relecture et de pratiques discursives qui peuvent nous donner des pistes fort intéressantes pour mieux comprendre la réception de «textes" télévisuels. Les fanfictions (ou fanfics ou fics, dans le jargon des fandoms) sont tous ces textes qui sur des supports et des formats différents (nouvelles, courtsmétrages, BDs, chansons, etc.) s'appuient sur les univers diégétiques de séries pour créer d'autres histoires ou explorer de possibilités narratives non développées dans le texte de base. La fanfiction constitue à cet égard un objet d'étude assez particulier, car elle témoigne autant d'une pratique discursive que d'une pratique de lecture et réécriture.

Notre travail essaie d'aborder les particularités et limites de cette réappropriation dans le cas précis des fanfics écrites pour la série policière américaine The Shield. Le choix de cette série n'est pas gratuit: The Shield est une série policière connue pour mettre au centre de son récit feuilletonesque une image des pouvoirs publics assez éloignée de ce qu'est la justice, le sens moral. L'intérêt est de voir jusqu'à où les fanfics produites à partir de la série poussent dans cette représentation. Notre travail essaie de tenir compte de la nature textuelle des fanfics pour décrire leurs rapports avec la forme du texte télévisuel de base. Il s'agit ainsi de préciser la forme textuelle de la réappropriation: les formes narratives développées et les formes énonciatives privilégiées.

\section{AUTEUR}

\section{MIGUEL-ANGEL TORRES}

Doctorant LARA, équipe CPST, Université Toulouse II Le Mirail 